
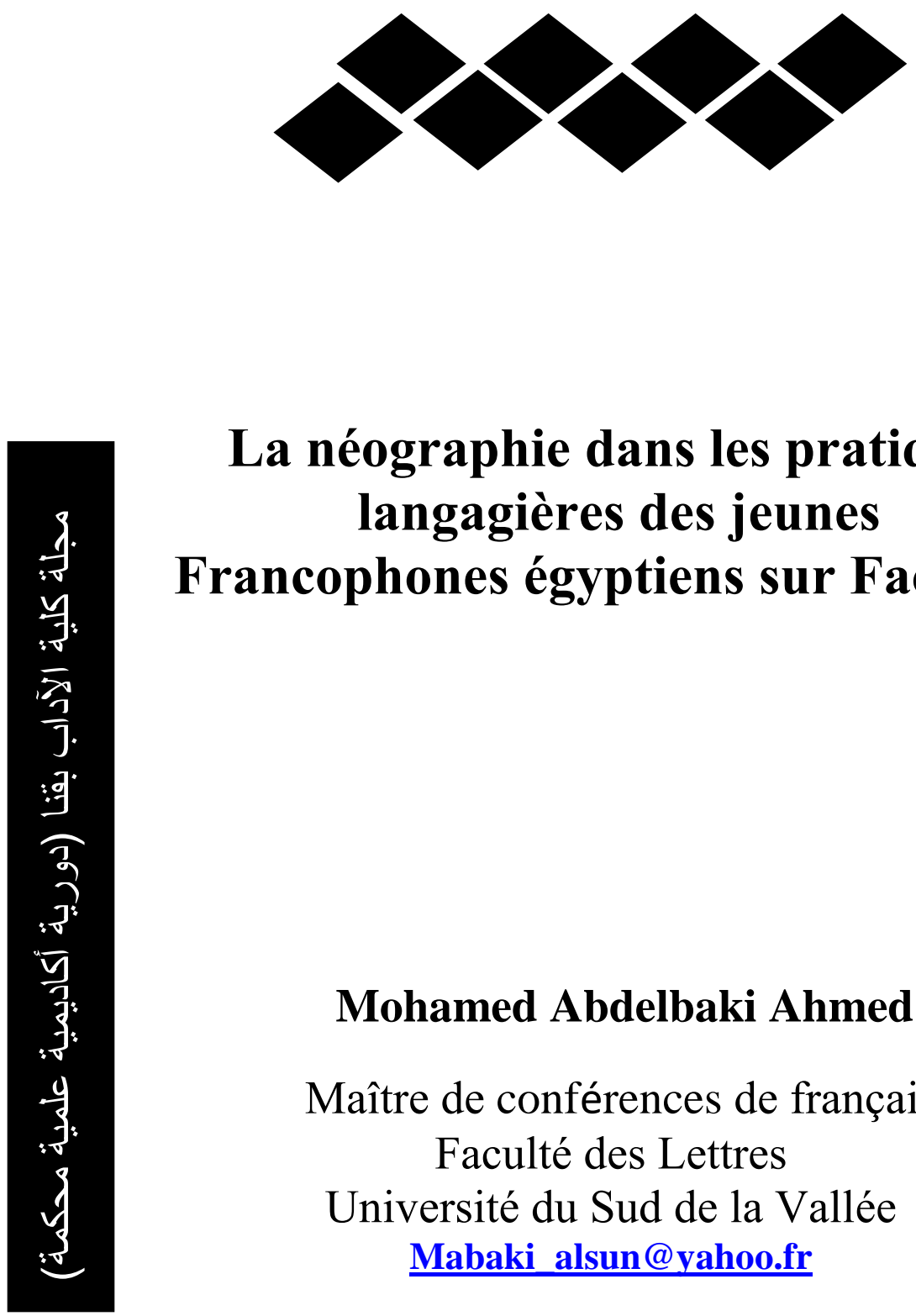

\title{
La néographie dans les pratiques langagières des jeunes Francophones égyptiens sur Facebook
}

Mohamed Abdelbaki Ahmed

Maître de conférences de français

Faculté des Lettres

Université du Sud de la Vallée

Mabaki_alsun@yahoo.fr 
Mohamed Abdelbaki Ahmed 


\section{Résumé}

Les dernières années du XXème siècle ont assisté au développement des technologies modernes de l'information et de la communication surtout l'emploi massif de l'Internet qui a déjà gagné tous les secteurs de l'activité humaine et sociale. Cette effervescence technologique a permis à l'homme de communiquer avec l'autre à l'échelle locale et internationale. Avec le temps, nous assistons à la naissance de nouveaux réseaux de communication sociale : Facebook, Twitter, et Instgram etc.,

L'objectif de la présente recherche est d'étudier ce phénomène puis expliquer les raisons de l'adoption d'un tel langage de la part des jeunes et énumérer aussi les formes de cette néographie à travers des exemples précis des publications des jeunes francophones égyptiens qui surfent sur Facebook. Notre groupe cible est constitué majoritairement de jeunes égyptiens en particulier les étudiants francophones de la Faculté des Lettres et de pédagogie de l'Université du Sud de la Vallée pour évaluer et préciser les grands points du phénomène. Le corpus choisi est le statut personnel de ma page sur Facebook tout en prenant des captures d'écran des publications de mes amis qui utilisent cette manière graphique soit en français soit en arabe. Dans les discussions des jeunes francophones, nous constatons la présence intense de la néographie non seulement dans des mots français mais aussi dans des mots arabes et anglais, ce qui souligne que les francophones sont influencés par les autres langues parlées en Egypte soit la langue maternelle soit la première langue étrangère en Egypte(l'anglais).

Dans cette étude, nous présentons les formes de cette néographie comme l'apocope, l'aphérèse, l'abréviation, les étirements graphiques, le rebus typographique... 


\section{Introduction}

Nous pouvons dire que les années 1990 ont assisté au développement des technologies modernes de l'information et de la communication surtout l'emploi massif de l'Internet qui a déjà gagné tous les secteurs de l'activité humaine et sociale. Cette effervescence technologique a permis à l'homme de communiquer avec l'autre à l'échelle locale et internationale. Avec le temps, nous assistons à la naissance de nouveaux réseaux de communication sociale : Facebook, Twitter, et Instgram etc., dont le langage se caractérise par des particularités lexicales et graphiques propres aux différents groupes et catégories socioprofessionnels. Les jeunes en général et les Egyptiens en particulier utilisent et fréquentent quotidiennement puis abondamment ces réseaux et applications de communication sociale, ce qui les a invités à adopter une nouvelle forme d'écriture intitulée: Cyberlangage, langage SMS, langage texto ou langage des tchatchs ${ }^{1}$. Ces différentes appellations désignent à peu près la même chose de l'écriture contemporaine chez les jeunes du monde entier. Ce phénomène nous pousse à traiter ses particularités lexicales et graphiques dans l'emploi quotidien de ces jeunes. Le nombre des jeunes qui accèdent au Facebook augmente régulièrement et le nombre total des égyptiens qui l'utilise arrive environ à 40 millions utilisateurs ${ }^{2}$, ce qui compromet et réserve un avenir linguistique hypothétique à la langue française et arabe, puisque ce langage juvénile ne respecte pas les normes de l'orthographe standard du français et de l'arabe. On constate le mélange aléatoire des codes linguistiques et l'utilisation de symboles graphiques qui permet à ces jeunes de s'exprimer rapidement et librement.

Nous pouvons dire qu'il y a deux formes d'interactions électroniques écrites : celles du temps réel c'est-à-dire synchrone où, les participants réagissent en même temps, échangent sur des sujets réciproques sans barrières temporelles, c'est la messagerie instantanée, Tchatch, etc., mais la deuxième forme s'intitule interactions asynchrones où les discussions entre les interlocuteurs en ligne sont différées et subissent un décalage temporel qui fait retarder le processus de l'émission et de la réception communicatives. Cela se remarque dans les blogues et les courriers électroniques.

L'objectif de la présente recherche est d'étudier ce phénomène puis expliquer les raisons de l'adoption d'un tel langage de la part des 
jeunes et énumérer aussi les formes de cette néographie à travers des exemples précis des publications des jeunes francophones égyptiens qui surfent sur Facebook. Pour collecter les données empiriques, nous avons recouru à la grille d'observation et surtout aux questionnaires. Notre groupe cible est constitué majoritairement de jeunes égyptiens en particulier les étudiants francophones de la Faculté des Lettres et de pédagogie de l'Université du Sud de la Vallée pour évaluer et préciser les grands points du phénomène. Le corpus choisi est le statut personnel de ma page sur Facebook tout en prenant des captures d'écran des publications de mes amis qui utilisent cette manière graphique soit en français soit en arabe. Nous avons également consulté le groupe des jeunes de l'Université intitulée les Francophones du Sud de la Vallée où les étudiants et les diplômés francophones de l'université discutent et échangent librement sans contraintes administratives ou formelles. Dans ce groupe, nous constatons la présence intense de la néographie non seulement dans des mots français mais aussi dans des mots arabes et anglais, ce qui souligne que les francophones sont influencés par les autres langues parlées en Egypte soit la langue maternelle soit la première langue étrangère en Egypte(l'anglais).

Avant d'étudier ces pratiques langagières dans le parler des jeunes égyptiens sur Facebook, nous allons d'abord faire un état des lieux sur la situation linguistique en Egypte pour que l'on sache la nature linguistique de la société où la néographie est présente dans les pratiques langagières de ses locuteurs et comment cette néographie constitue un danger pour l'avenir de la langue française et arabe et ensuite pour la production littéraire et intellectuelle en l'Egypte.

\section{La situation linguistique en Egypte ${ }^{3}$}

En Egypte, l'arabe est la langue officielle, mais comme les autres pays arabes, une situation diglossique se présente entre l'arabe standard et l'arabe dialectal, celui-ci est influencé par le contact et la proximité linguistique avec d'autres langues étrangères sur le territoire égyptien. Il s'agit par exemple du français, de l'anglais, de l'allemand, de l'italien etc. Le dialecte d'Egypte reste toujours varié d'après le lieu géographique et la situation éducative et professionnelle des locuteurs. Le dialecte du Caire représente chez quelques locuteurs le parler des 
jeunes souhaitant accéder à une situation de prestige et de promotion sociale. Cette qualité acquise à la variante orale du Caire est due à l'adoption de ce dialecte dans les feuilletons et le cinéma : "L'une des variantes $d u$ dialectal, celle du Caire, devient la variante orale de prestige dans les frontières d'un Etat fortement jacobin (...), mais audelà de ces frontières par le moyen des films et des séries télévisées, les musalsalât $t^{4}$. Nous observons aussi dans le parler quotidien des locuteurs égyptiens que le dialectal passe d'un statut oral à un statut écrit dans les pratiques langagières des jeunes sur l'internet, dans les enseignes des magasins, dans les quelques articles de la presse etc. Bien que l'arabe domine le parler quotidien d'Egypte, il y a d'autres langues parlées par des groupes minoritaires qui sont loin de tout contrôle gouvernemental comme le dorami, le nubien, le berbère et le beja. Ces langues sont pratiquées par des communautés bien identifiées ethniquement et associées à des espaces propres et à une histoire ancienne ${ }^{5}$.

Le nubien est une langue parlée par un groupe d'habitants à Assouan. Avant la construction du haut barrage cette langue se parlait par deux communautés les Kenzi, au nord, et les Faddica au sud ${ }^{6}$. Ces communautés linguistiques ont bien changé leurs habitudes langagières et ont appris l'arabe à la suite de leur déplacement et leur intégration en Egypte et elles sont devenues bilingues et par conséquent l'arabe devient leur langue maternelle.

Le berbère se rencontre dans les oasis égyptiennes de Siwa à la frontière de la Libye. Les enfants de ces régions n'apprennent l'arabe qu'à l'école. Le siwi a déjà emprunté un nombre considérable de mots à l'arabe. Le beja se parle dans l'extrême Sud de l'Egypte aux frontières soudanaises et dans la Vallée du Nil d'Assouan à Daraw. Ses locuteurs sont bilingues beja-arabe. Le dorami ou le tzigane d'origine indoiranienne, se parle par des groupes divisés en deux. Les Ghagar en Basse-Egypte, et Halabi en Haute-Egypte ${ }^{7}$. Ces communautés provenant d'espaces géographiques lointains ne sont pas douées d'une valorisation sociale chez les familles égyptiennes.

Bref, l'arabe dialectal d'Egypte regroupe quatre types: l'arabe égyptien qui englobe le dialecte Cairote (le plus prestigieux de tous les dialectes en Egypte) dont le nombre des locuteurs est nombreux et augmente régulièrement, l'arabe Saïdi parlé dans les régions de la 
Haute-Egypte, l'arabe bédouin occidental parlé à l'ouest d'Alexandrie à côté de la frontière de la Libye, l'arabe bédouin de Sinai ${ }^{8}$. Mais en Egypte nous constatons que les dialectes les plus courants sont le dialecte cairote et le dialecte Saidi comme le dit Catherine Miller :

"Il n'y a donc pas une unité dialectale mais tous les dialectes de Haute-Egypte se distinguent assez radicalement du parler du Caire... Les cairotes comprennent très mal les dialectes de Haute-Egypte et en ont une vision très stéréotypée et souvent péjorative, alors qu'à l'inverse les Sa'aidas comprennent le dialecte du Caire et ne le parle pas $^{9} . "$

Mais pour les langues étrangères présentes en Egypte, nous constatons que l'anglais représente la première langue étrangère puisqu'elle est considérée comme la langue de mondialisation et du commerce international. La langue française est classée deuxième langue étrangère au secteur public et privé (on ne parle pas des écoles privées de langues) et elle est enseignée dès la première année du secondaire en concurrence avec d'autres langues étrangères comme l'allemand, l'italien, l'espagnol. Le choix du français de la part des étudiants à l'étape secondaire comme deuxième langue étrangère dépasse celui des autres langues européennes. A l'université, l'enseignement des langues étrangères s'observe au sein des Facultés des Lettres et de Pédagogie mais souvent avec une priorité particulière à l'anglais et au français, et aux facultés des langues ( Al Alsun) l'enseignement de la plupart des langues du monde est présent.

\section{Le Facebook et le langage des jeunes}

Le Facebook représente le plus grand réseau social à l'échelle mondiale. Ce réseau inventé par Mark Zuckerberg pour un but académique propre mais avec le temps Mark a développé son réseau à l'aide de deux autres étudiants de la même université pour lancer une nouvelle version sous le nom de Facebook qui a été acheté en 2005 pour un montant 200,000 dollars américains. Ce réseau, à but communicatif, donne la chance à ses utilisateurs de publier et de partager des publications, des photos, des opinions afin de contacter des amis réels ou virtuels et aussi pour discuter des questions du travail 
ou de famille. Le Facebook aide à renouer, réactiver des échanges culturels et promouvoir l'interaction avec les autres.

Les jeunes égyptiens sont le noyau de la société. Ils adoptent dans leur parler quotidien des pratiques langagières propres et ils ont la pleine liberté de s'exprimer sur les réseaux sociaux, d'où nous observons un langage qui dévie des normes de la langue dans le système phonologique, morphologique, et sémantique.

La néographie est considérée comme une nouvelle forme d'écriture composée de procédés multiples de créativités linguistiques qui ne correspondent jamais aux normes de l'écriture standard de la langue. Déjond souligne que la néographie représente une langue remplie d'abréviations, de sigles, de rébus de troncations qui sont de nature à transformer la forme scripturale de la langue ${ }^{10}$. D'ailleurs, J. Anis souligne qu'il y a deux procédés orthographiques de la néographie : les premiers comportent les troncations, les anglicismes et les onomatopées puis le verlan, le deuxième regroupe les graphies phonétisantes, les rébus à transfert, les logogrammes et les étirements graphiques $^{11}$. Dans le langage des jeunes francophones d'Egypte, nous pouvons lire le mot bonjour en BJR, pourquoi peut s'ecrire pkoi ou $p k$, l'adverbe bien peut s'écrire $B 1$, l'expression Inshallah peut-être abrégé en ISA. Bref, l'emploi de "cette néographie fait apparaître une nouvelle graphie pour un signifiant déjà existant dans la langue ${ }^{12 ",}$ c'est-à-dire les locuteurs jeunes écrivent d'une nouvelle manière qui ne correspond pas aux règles traditionnelles orthographiques de la langue. Dans ce langage les formules "d'ouverture et de clôture sont réduites, les abréviations et le style télégraphique sont utilisés, la ponctuation standardisée est omise, les accents et les majuscules en début de phrases sont absentes ${ }^{13}$."

D'autre part, pour les raisons de l'emploi de la néographie chez les jeunes, des chercheurs et des critiques présentent leurs points de vue à l'égard de ce phénomène:

1- Safwat Al- Allem voit que la parution d'un nouveau langage chez les jeunes représente une chose banale qui se répète régulièrement. Selon lui, ce phénomène reflète la rébellion sociale des jeunes et l'absence de l'interaction avec les personnes âgées. Il indique que l'internet n'est pas le seul responsable de ce problème mais aussi l'emploi massif de l'anglais et la détérioration de l'enseignement de 
l'arabe en plus le drame égyptien qui présente des feuilletons et des films contenant un nombre considérable d' expressions et de termes bizarres ${ }^{14}$.

- Ghassane Mourad ${ }^{15}$ affirme que l'emploi de ce langage dans les discussions instantanées des jeunes arabes sur Facebook arrive avec l'introduction des moyens technologiques modernes comme l'ordinateur et le téléphone portable qui ne contenaient qu'un clavier anglais, ce qui a obligé les utilisateurs à trouver une autre alternative graphique pour la communication et l'échange sur les réseaux sociaux. Il souligne également que ce processus a continué même après la disponibilité d'un clavier arabe. Selon lui, l'emploi du langage des jeunes ne se limite pas aux discussions instantanées sur l'internet mais ce langage a pénétré le vif de la vie quotidienne puisque les jeunes l'utilisent dans le parler quotidien sans hésitation, ce qui traduit la nature audacieuse des jeunes qui veulent tout imiter pour devenir distingués des autres à travers un code linguistique propre ${ }^{16}$.

- Ali Salah ${ }^{17}$, a mis en garde le langage des jeunes puisque celuici menace l'avenir de la langue arabe et il peut conduire à des conséquences néfastes sur la culture de la langue. Ses résultats sur un échantillon des jeunes âgées de 15 à 35 ans, ont montré le rôle négatif de l'Internet, caractérisé comme un moyen de communication facile et rapide sur l'arabe contemporain puisque les jeunes ont commencé à insérer les chiffres et les symboles au sein de l'alphabet arabe, le chiffre 7 remplace celle de $h$, le chiffre 3 remplace celui de $\varepsilon$, le coup de glotte se remplace par $2 \ldots{ }^{18}$. D'après lui, les jeunes recourent à l'emploi intense de ce langage à cause d'un sentiment croissant de l'aliénation qui les pousse à la rébellion sociale par la formation d'un monde virtuel à l'aide de ce langage énigmatique pour les autres et considéré comme une masque face aux personnes âgées.

- Dans un article intitulé le phénomène de l'arabe hybride touche l'arabe d'une monstruosité linguistique, Nahla Elnemr indique que ce langage a produit centaines de blogues, des textes en prose et les jeunes justifient le choix de ce type du langage au manque de logiciels qui ne supportent pas l'emploi de l'arabe. Selon elle, ce fait est lié à l'emploi de la langue anglaise qui est la première langue dans les réseaux sociaux et dans la technologie moderne, ce qui les a encouragés à 
préférer ce langage aux dépends de la langue maternelle. Elle prévoit que ce langage ne subsistera pas longtemps parce qu'il n'a pas de règles fixes et il est restreint à une classe socialement riche et dont le nombre est limité par rapport aux autres couches sociales. Nahla Elnemr souligne que le danger de ce phénomène réside au fait de sa transmission aux medias visuelles et en particulier les programmes de

divertissement destinés aux jeunes, ce qui est un indice très préjudiciable pour l'avenir de l'orthographe et de la langue. De surcroît, le malheur est que, les compagnies des technologies modernes disposent des programmes consacrés à la facilitation de l'emploi de ce langage comme les applications électroniques: Marine et francotranslate.com, etc., ce qui constitue une menace directe pour la langue et la culture arabes ${ }^{19}$.

De tout ce qui précède, nous comprenons que ce phénomène n'est pas limité à la langue française mais également il touche la langue arabe et anglaise et nous pouvons résumer les raisons communes qui invitent à l'utilisation de la néographie chez les jeunes égyptiens en général et les francophones en particulier, ceux-ci mélangent dans leurs conversations sur Facebook des codes linguistiques différents:

- la détérioration de l'apprentissage de la langue soit maternelle ou étrangère a influencé négativement le niveau linguistique des jeunes et les a encouragé à adopter un langage hybride qui échappe à tout contrôle linguistique.

- l'absence d'un clavier arabe et l'indisponibilité de logiciels ont encouragé les jeunes égyptiens à recourir aux caractères latins, et cela s'observe abondamment chez les jeunes arabes expatriés en pays étrangers représentant leur "ingéniosité dans l'appropriation de l'invention face aux difficultés" et par conséquent la domestication d'une ressource ${ }^{20 "}$ ou à vrai dire ce procédé entre dans "la catégorie des mécanismes d'appropriation de l'Internet dans le monde arabe ${ }^{21 "}$. Pour cela les jeunes reproduisent de nouvelles formes composées des lettres et des chiffres pour représenter quelques phonèmes ou sons absents du clavier latin ${ }^{22}$.

- La fascination des jeunes de tout ce qui est étranger a créé chez eux un sentiment d'infériorité à l'égard de la langue standard. La conséquence est qu'ils ont été obligés d'adopter de nouvelles habitudes 
La néographie dans les pratiques langagières des jeunes Francophones égyptiens sur Facebook

linguistiques qui peuvent être qualifiées de bizarres puisque cette néographie inventée n'est pas présente ni dans la langue d'étude ni dans une autre langue étrangère.

Formes de la néographie chez les francophones égyptiens

\section{1-1- La troncation}

La troncation est une abréviation d'un mot par suppression de certains phonèmes. L'aphérèse et l'apocope sont les deux formes de la troncation :

\section{- l'aphérèse :}

C'est le retranchement d'une syllabe ou d'une lettre au début d'un mot. Nous trouvons cette chute initiale de la lettre dans des mots utilisés dans le langage Facebook de la part des francophones égyptiens. Nous avons trouvé peu d'exemples des mots français dans le langage des jeunes sauf le mot autobus qui devient bus, le mot petit qui peut être abrégé en $\boldsymbol{t} \boldsymbol{i}$, mais nous avons observé dans ce groupe des Francophones du Sud de la Valle que les étudiants peuvent pratiquer l'aphérèse dans des mots arabes qu'ils répètent entre eux comme dans les mots /?ahabibi/(mon amour),/?asahbi/( mon ami) /?asadiqi/(mon ami), où les locuteurs égyptiens réduisent l'outil d'apostrophe arabe /ya/ en $/ \boldsymbol{a} /$ seulement et ils suppriment la lettre $/ \boldsymbol{y} /$ des mots déjà mentionnés. Nous observons également que l'outil d'apostrophe est tronqué et il se transforme en hamza de coupure pour remplacer complètement le procédé d'apostrophe d'arabe standard. Cette aphérèse atteste un changement partiel dans la graphie et elle marque la fluidité du discours des jeunes qui peut adopter n'importe quelle forme sous prétexte de créativité lexicographique et cela dénote également le niveau faible des locuteurs francophones de ce groupe qui adopte des mots arabes, ce qui souligne que la question de l'écriture normative en français n'est pas le but espéré mais seulement l'acte communicatif entre locuteurs est le but essentiel.

\section{- l'apocope}

On appelle apocope un procédé courant d'abréviation consistant à supprimer les syllabes finales d'un mot polysyllabique ${ }^{23}$ L'apocope est le contraire de l'aphérèse. Elle est une élision d'un ou plusieurs phonèmes en fin de mot. Dans le langage Facebook, l'apocope s'emploie abondamment plus que l'aphérèse. Voici des exemples ou les 
locuteurs francophones suppriment les dernières lettres des mots dans leurs échanges langagiers:
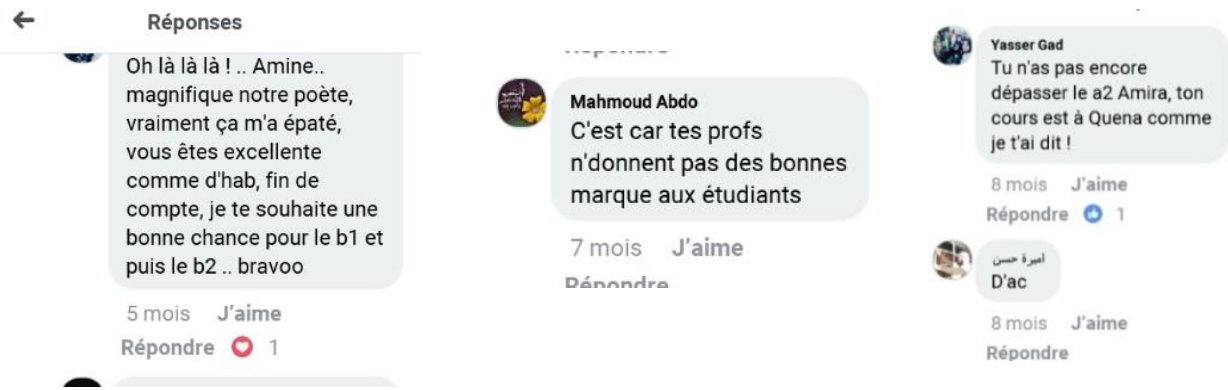

Dans les trois exemples mentionnés ci-dessus, le premier locuteur supprime la fin du mot habitude dans l'expression comme d'habitude, tandis que le deuxième locuteur supprime les dernières lettres du mot professeur qui devient prof, et le troisième énonciateur remplacent l'adverbe d'accord en d'ac. Ce qui montre que les francophones égyptiens cherchent l'emploi des mots tronqués et rapides et la question d'écrire en orthographe standard n'est pas considérée comme une chose de grande valeur. De plus, nous pouvons dire que les locuteurs francophones utilisent également l'apocope pour d'autres mots français et anglais dans leurs pratiques langagières. Voici des exemples:

\begin{tabular}{|c|c|c|}
\hline Le mot apocopé & Le sens en français & Le mot avant l'apocope \\
\hline Admin & administrateur & Administrator \\
\hline Doc & docteur & Docteur/ \\
\hline bro & frère & Brother \\
\hline Tel & téléphone & Téléphone \\
\hline Tab & tablette & Tablette \\
\hline pic & photo & Picture \\
\hline prof & professeur & Professeur \\
\hline Fac & Faculté & Faculté \\
\hline foot & football & Football \\
\hline photo & photographie & Photographie \\
\hline D'ac & D'accord & D'accord \\
\hline M & Monsieur & Monsieur \\
\hline Comme d'hab & Comme d'habitude & Comme d'habitude \\
\hline
\end{tabular}


Dans les exemples ci-dessus, nous remarquons que les jeunes peuvent abréger à leur gré le mot français ou anglais. Cette apocope touche des mots, des expressions et des adverbes. Les internautes francophones en Egypte peuvent appliquer la même règle de l'apocope pour des prépositions arabes ${ }^{24}$. Nous observons que le langage des jeunes sur Facebook se distingue par le plurilinguisme et nous trouvons, comme on le verra plus tard, aussi une alternance codique où le rébus typographique est encore fréquent.

Bref, L'aphérèse et l'apocope se croisent beaucoup dans le langage des jeunes sur Facebook et cette nature de la troncation ne peut pas être comprise par une personne âgée ou qui se passionne par l'orthographe classique, ce qui marque une particularité lexicographique de ce langage des jeunes.

\section{Les acronymes et les abréviations}

L'acronyme est un sigle qui aide à prononcer des lettres comme un mot ordinaire sans épeler ${ }^{25}$. Les Francophones égyptiens écrivent facilement $M D R$ au lieu de mourir de rire, stp au lieu de s'il te plait, Sms au lieu de short message service, Lol qui signifie qu'une personne rit à haute voix. Egalement, nous observons qu'ils utilisent l'abréviation qui consiste à retrancher des lettres du mot ou d'un syntagme afin de le raccourcir pour écrire plus vite ou économiser de l'espace ${ }^{26}$. L'article de l'interrogation pourquoi se transforme en pkoi. Nous constatons que l'expression arabe Inshallah devient ISA, et Mashallah se transforme en $M S A$. ils appliquent la même règle pour des mots anglais qu'ils emploient énormément comme thx au lieu de thanks, la préposition with peut s'écrire $w z$, please peut être abrégé en plz. Les Francophones d'Egypte sur Facebook peuvent aussi réduire le mot à des squelettes consonantiques c'est-à-dire ils suppriment les voyelles des mots et ils choisissent les consonnes principales des mots ${ }^{27}$. Ce phénomène est visible dans des mots comme le mot facebook qui devient $f b$, tout devient $t$, bonsoir est abrégé en bsr, l'adjectif indéfini quelque devient $k k$, les adverbes toujours et beaucoup deviennent tjr et bcp. Aussi ils peuvent appliquer la même règle de l'abréviation pour des mots arabes: Wallahi qui se transforme en Wlhi, le mot Tayeb qui devient Tyb, le mot Keda qui devient kda, le mot habibi devient aussi hbbi. Parfois nous remarquons que les francophones égyptiens peuvent dire 
facilement $d s l$ au lieu de désolé, $m t n$ au lieu de maintenant. Ils réduisent également le mot à des squelettes vocaliques comme nous le remarquons dans la voyelle $u$ qui remplace le pronom you en anglais et l'expression how are you qui devient $h r u$, you are welcome se transforme en $\mathrm{Yr}$ welcome. Nous constatons que les francophones d'Egypte peuvent adopter plusieurs codes linguistiques dans leur interaction et ils appliquent la même règle d'acronyme et d'abréviation pour des mots français, arabes et anglais, ce qui traduit l'inégalité des compétences linguistiques des locuteurs francophones à l'égard de l'emploi des mots et des expressions. Pour cela ils recourent à des mots anglais et arabes pour remplir les cases vides des mots français absents de leur lexique quotidien.

\section{Les anglicismes}

Les internautes égyptiens en général, et les Francophones d'Egypte en particulier utilisent des mots d'origine anglaise soit dans leurs discussions instantanées, soit dans leurs publications soit dans leurs commentaires. Cet emploi des mots anglais montre l'importance et le prestige de la langue anglaise sur l'échelle mondiale due à l'invasion culturelle puis scientifique de l'Angleterre et de l'Amérique dans le monde francophone et arabe. Ces mots appartiennent à tous les domaines de la vie quotidienne et les locuteurs emprunte à l'anglais tous les éléments de la langue comme les noms, les verbes, les adjectifs, les adverbes, les prépositions etc. En voici des exemples:
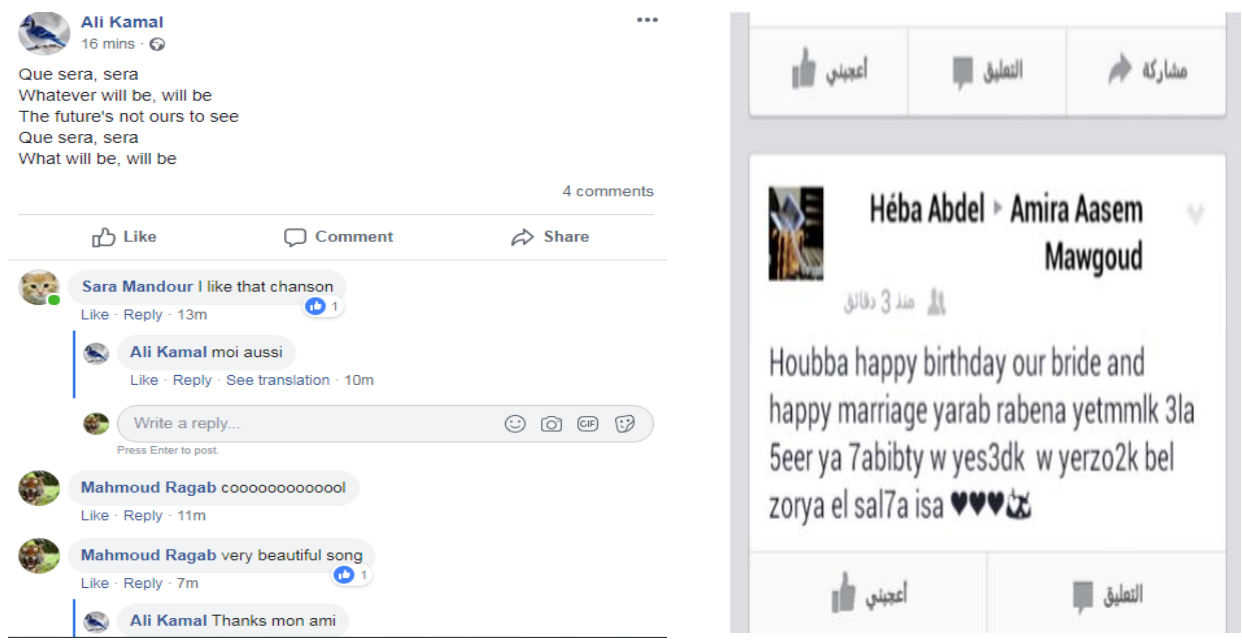
Le premier exemple comporte des mots et des phrases écrites en anglais. Le locuteur répète les mots de la chanson populaire : Que Sera Sera, écrite en 1956 par l'équipe de Jay Livingston et Ray Evans. Le premier locuteur répond en anglais mais aussi il ajoute le mot français chanson. Le deuxième locuteur emploie le mot cool pour exprimer son impression de la chanson et le troisième locuteur écrit une phrase complète en anglais et celui qui a publié les mots de la chanson exprime en français hybride ses remerciements: Thanks, mon ami. Cet emploi des mots anglais dans l'interaction verbale des internautes francophones égyptiens montre que l'anglais est encore présent dans leur échange langagier pas seulement par des mots mais aussi par les phrases qui représentent, comme on le verra ci-dessous, une forme d'alternance codique. Le deuxième exemple comporte une phrase complète en anglais: Happy birthday our bride and Happy marriage. La locutrice emploie également des mots arabes écrits en caractères latins tout en utilisant le rébus typographique qui mélange les symboles et les chiffres, en outre, une abréviation du mot inshallah en ISA. Ce qui souligne que les locuteurs et les destinataires du Facebook peuvent communiquer en employant des mots et des expressions en langues différentes et peu importe la question de l'identité linguistique. Il nous parait à travers les deux exemples ci-dessus que les locuteurs expriment avec toutes sortes de liberté leurs idées et leurs souhaits. L'emploi des mots anglais à côté des mots français et arabes souligne une intégration suprême de l'anglais dans le discours des utilisateurs des réseaux du contact social. Aussi, l'emploi des mots arabes en caractères latins signifie que les locuteurs égyptiens en général se passionnent pour l'emploi des langues étrangères et la question de l'emploi de l'orthographe normatif, soit en français ou en arabe, n'est pas tenue dans leur compte.

\section{L'alternance codique}

L'alternance codique permet au locuteur de passer d'une langue à une autre dans la même conversation et elle représente la juxtaposition à l'intérieure de la même discussion entre locuteurs des passages dont le système linguistique est tout à fait différent ${ }^{28}$. L'alternance codique peut avoir d'autres synonymes comme le discours mélangé $e^{29}$, rencontre $d^{\prime}$ habitudes linguistiques ${ }^{30}$, le heurt de deux langues ${ }^{31}$. Nous pouvons distinguer deux types d'alternance codique, l'une conversationnelle, 
l'autre situationnelle. Le premier type revoie à l'emploi de deux variétés linguistiques dans la même conversation. Ce type se pratique dans les discussions quotidiennes et familières et nous ne l'observons pas dans les échanges formels. Gumperz souligne que les locuteurs, dans ce type, doivent avoir des codes et de principes d'interprétations communs et cela "prend la forme des présupposés tacites, acceptés comme allant de soi, que recouvre le mieux l'analyse indirecte de la conversation ${ }^{32 " .}$ Le deuxième type intitulé, l'alternance codique situationnelle, qui s'attache à une activité professionnelle ou au contact d'un interlocuteur positionné dans une catégorie sociolinguistique différente de celle du locuteur $^{33}$. Ce type d'alternance n'est pas habituel et dépend de la situation de la communication. Dans le langage des jeunes sur Facebook, nous constatons l'abondance de cette alternance codique du type conversationnel qui touche la structure interne du langage des jeunes. Le locuteur peut aisément transformer le code linguistique sans aucune difficulté de la part du récepteur qui le comprend et qui continue à parler dans un code linguistique tout à fait différent d'un troisième interlocuteur qui commente ou parle dans un code différent aussi mais aussi compréhensible. Le degré de compréhension entre interlocuteurs compte sur le répertoire linguistique et la connaissance des langues étrangères puisqu' il est impossible pour un locuteur de codifier sa parole ou ses commentaires sans l'admission implicite de l'autre avec qui l'échange linguistique se passe dans un climat qui se distingue par la compréhension réciproque des codes avec l'accord de tous les interlocuteurs de la conversation. Parfois nous trouvons plusieurs formes d'alternance codique:

\section{- l'alternance intra-phrastique}

Dans cette forme, nous remarquons la présence de deux structures syntaxiques différentes dans la même phrase. Poplack souligne que le locuteur dans la même conversation peut employer des structures syntaxiques qui appartiennent à deux langues différentes au sein de la même conversation ${ }^{34}$. Voici deux exemples concrets : 
La néographie dans les pratiques langagières des jeunes Francophones égyptiens sur Facebook

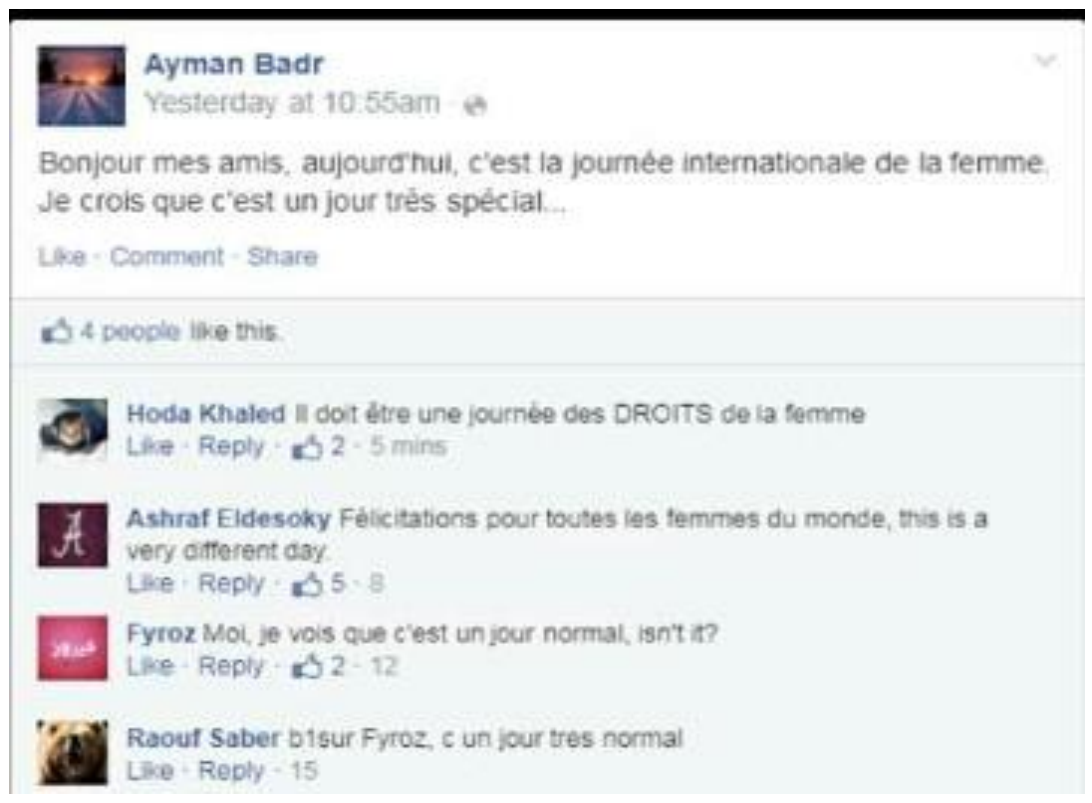

Dans l'exemple cité ci-dessus, le locuteur informe les interlocuteurs de la journée internationale de la femme. Les interlocuteurs échangent les félicitations et les bons vœux pour l'avenir de la femme. Nous remarquons que le deuxième locuteur utilise deux codes linguistiques différents: le français et l'anglais dont le système syntaxique et morphologique n'est pas le même. Le troisième locuteur exprime en français son point de vue à l'égard de cette journée mais à la fin de sa phrase il pose une question en anglais isn't it? ce qui souligne que les locuteurs bilingues peuvent changer facilement le code linguistique mais toujours avec l'admission implicite des destinataires, qui se transforment en locuteurs principaux quand ils expriment en codes différents leurs points de vue a l'égard du sujet abordé. Bref, cette forme d'alternance codique se trouve abondante dans la plupart des discours des bilingues qui maîtrisent la syntaxe et la sémantique de deux langues.

\section{L'alternance codique inter-phrastique}

Cette forme correspond à l'emploi alternatif au niveau d'unités plus longues, de phrases ou de fragments de discours, dans la production d'un même locuteur ou dans les prises de paroles entre interlocuteurs ${ }^{35}$. 
Voici deux exemples :
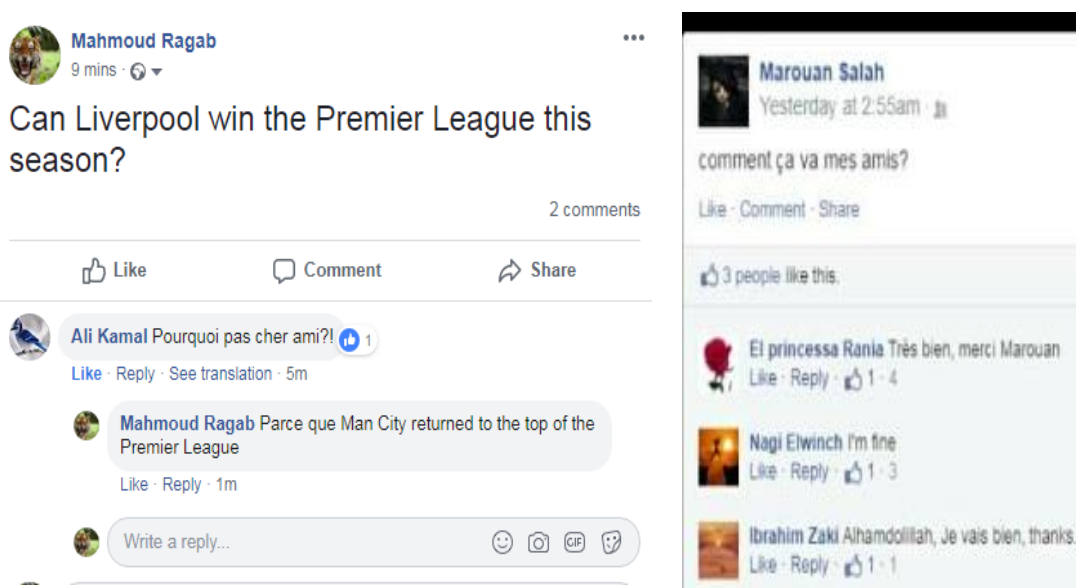

Dans les deux exemples mentionnés ci- dessus, nous observons que les locuteurs du premier exemple échangent des codes en français et en anglais. Le premier locuteur pose une question en anglais à ses interlocuteurs mais il reçoit une réponse en français de la part du premier récepteur, et il répond aussi en anglais à la question de son ami tout en y insérant la conjonction de subordination de cause parce que en français puis des mots justificatifs en anglais, ce qui démontre que l'émetteur et le récepteur partagent les mêmes qualités de la connaissance linguistique et le processus de l'émission et de la réception du message se passe sans aucun malentendu de sens. Pour la deuxième photo, nous remarquons l'alternance codique interphrastique, représentée dans la prise des paroles entre interlocuteurs; la première locutrice répond en français à la question posée, et le deuxième locuteur répond en anglais, alors que le troisième locuteur utilise trois codes différents: arabe en caractères latins, français et anglais. Dans ce type d'alternance codique, nous observons la liberté des interlocuteurs de pratiquer le bilinguisme ou le trilinguisme avec l'accord implicite de tous les participants du cadre conversationnel. Tout cela montre que les locuteurs peuvent confondre des codes dans des phrases complètes ou à l'intérieure de la même phrase. Cette alternance codique ne peut pas être comprise par un autre locuteur qui ne partage pas les mêmes particularités juvéniles. 
La néographie dans les pratiques langagières des jeunes Francophones égyptiens sur Facebook

Parfois nous rencontrons des alternances codiques qui comportent sur trois codes linguistiques différents:

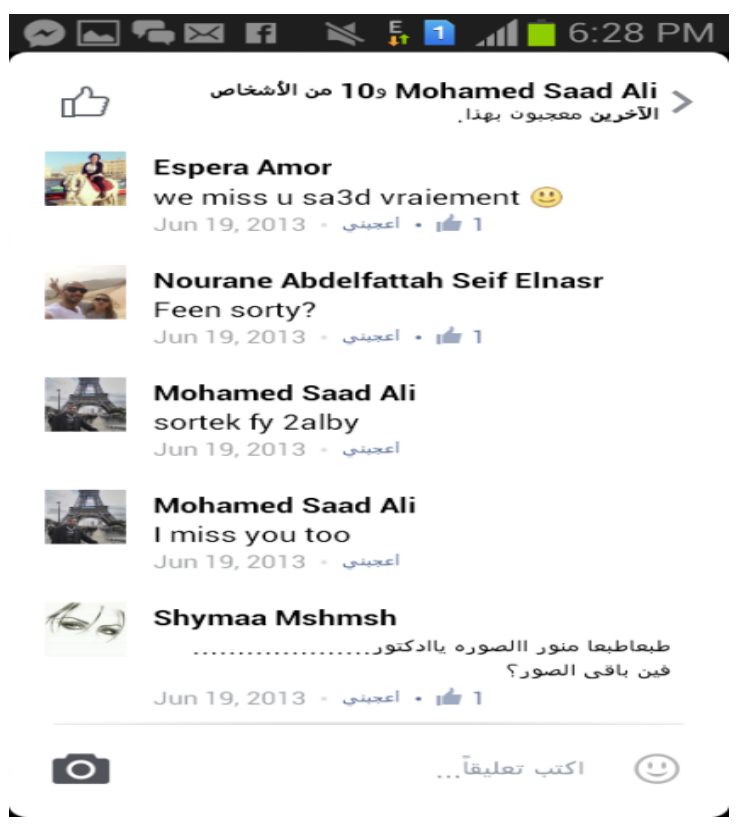

La première locutrice commence sa phrase en anglais puis elle utilise le rébus et à la fin de la phrase elle emploie l' adverbe (vraiment) en français, la deuxième locutrice pose une question en arabe mais en caractères latins, mais l'émetteur qui a déjà écrit la publication répond en employant deux codes différents : il répond en anglais à la première locutrice, et en arabe hybride pour la deuxième, ce qui signifie que les locuteurs peuvent échanger des codes linguistiques différents au sein de la même conversation sans aucune difficulté de la part de l'ensemble des interlocuteurs.

\section{- l'alternance codique extra-phrastique}

C'est l'emploi des expressions idiomatiques, des proverbes, des dictons dans des segments monolingues au sein de la conversation. Nous n'avons pas relevé d'exemples de ce type d'alternance extraphrastique dans la prise de paroles des jeunes sur Facebook.

De plus, nous avons observé d'autres types d'alternance codique dans les pratiques langagières des jeunes égyptiens :

- une alternance codique qui se produit entre des variétés dialectales de la même langue; le parler cairote n'est pas semblable au 
parler de la Haute-Egypte et cela se reflète évidemment dans les discussions des jeunes à l'université, celle-ci regroupe des étudiants issus d'endroits différents sur le plan social et linguistique ;

- une alternance codique entre l'arabe standard et l'arabe dialectal puisque le locuteur peut utiliser des codes linguistiques classiques et dans la même phrase il utilise des codes dialectaux.

Bref l'alternance codique s'observe évidemment dans le langage des jeunes sur Facebook et cette alternance codique ne peut pas être partagée par les autres puisque les jeunes ont leurs codes, leurs signes et leurs référents. Cette alternance codique est une particularité lexicale et graphique du langage des jeunes et elle forme un élément essentiel de la néographie chez les jeunes.

\section{Les rébus typographiques}

Le rébus est une suite de lettres, de mots, de dessins représenté par les sons qu'ils évoquent la syllabe, le mot ou la phrase que l'on veut deviner $^{36}$. Cette technique représente un jeu avec la langue et il consiste à faire deviner des mots en employant des dessins et des chiffres énigmatiques ou des signes particuliers pour les déchiffrer grâce à leur valeur phonétique.

En lisant les commentaires et les publications de mes amis, mes proches sur Facebook, je constate clairement l'emploi intense de ce phénomène qui mélange des chiffres et des lettres. Ce procédé typographique n'a pas de règles fixes et chaque internaute a son propre style dans la transcription de ses paroles vers l'emploi de mots métissés et compris par ses interlocuteurs. Ce langage où le rébus est utilisé énormément s'intitule le français hybride selon les locuteurs francophones et l'arabe hybride pour les internautes égyptiens; c'est-àdire l'emploi des mots arabes ou français en caractères latins en y insérant des chiffres et des symboles. Ce langage n'est pas formel et il est comme résultat de la technologie numérique que la société atteste il y a quelques années ${ }^{37}$. Les internautes égyptiens ont des chiffres particuliers pour chaque son utilisé: 
La néographie dans les pratiques langagières des jeunes Francophones égyptiens sur Facebook

\begin{tabular}{|c|c|}
\hline Le 2 pour /Í/ & Le 7 pour le / / \\
\hline Le 3 pour le /ع/ & Le 8 pour le /غ / \\
\hline Le 5 pour le / & Le 9 pour le \\
\hline Le 6 pour /b/ & \\
\hline
\end{tabular}

Mais pour les francophones en général, on a constaté qu'ils utilisent des rébus typographiques spécifiques et énigmatiques comme l'illustre le tableau suivant ${ }^{38}$ :

\begin{tabular}{|l|l|}
\hline Les rébus typographiques & Le sens en français \\
\hline B1 & bien \\
\hline Bn8 & Bonne nuit \\
\hline L8 & lui \\
\hline PL1 & plein \\
\hline QLQ1 & Quelqu'un \\
\hline PS 2 Koi & Pas de quoi \\
\hline 9 & neuf \\
\hline
\end{tabular}

Nous avons constaté que les locuteurs francophones égyptiens utilisent rarement les rébus typographiques pour des mots français dans leurs interactions conversationnelles. Nous avons relevé un seul exemple:

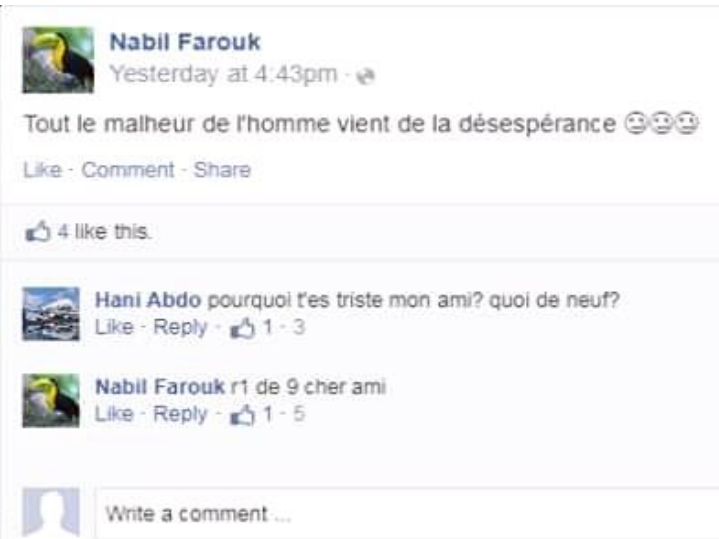

Ici le locuteur répond à son ami par ce langage du rébus où nous observons la fréquence des chiffres et des lettres qui forment des mots énigmatiques et décryptées de la part des interlocuteurs, ceux-ci utilisent le chiffre 1 au lieu de la syllabe ien, et le chiffre 9 au lieu de 
l'adjectif neuf. Cette codification des mots semble être une impasse linguistique pour les autres, qui ne maitrisent pas cette manière d'écriture. Mais nous avons trouvé que dans la plupart du temps les francophones appliquent les rébus typographiques pour des mots arabes qui s'insèrent couramment dans leurs interactions conversationnelles, ce qui démontre que leur niveau linguistique en français ne leur permet pas parfois de connaitre les nouvelles formes des rébus typographiques utilisés par les Internautes français de souche ou de francophones marocains ou africains. Voici des exemples où le rébus est employé en arabe mais toujours en caractères latins:

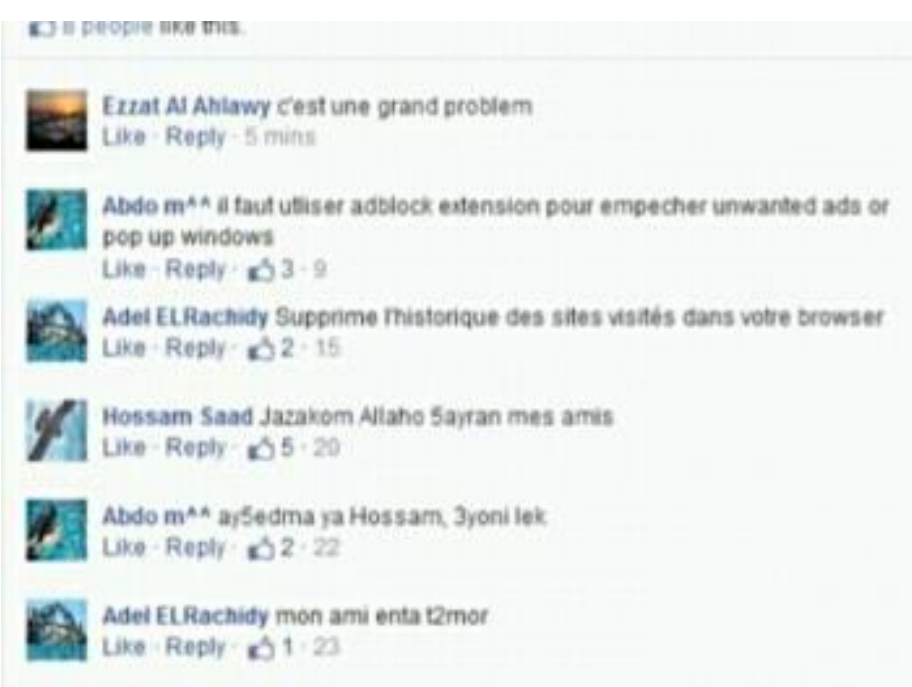

Dans la photo ci-dessus, les locuteurs appliquent les rébus typographiques pour des mots arabes, ce qui souligne que la question d'écrire en bon français n'est pas encore tenue en compte chez eux. Par conséquent, Les locuteurs excellent dans l'emploi des rébus typographiques pour des mots arabes dans une conversation en français. Pour un locuteur âgé ou un locuteur qui ignore ce langage, il devient très étonné de l'emploi de ces chiffres énigmatiques au sein des mots. L'auteur Ahmed Khaled Tawfik exprime son étonnement de ce langage qui réunit des chiffres et des symboles. Il dit que la première fois qu'il découvre ce langage quand il a reçu un message de son ami et il croyait que c'était une langue finlandaise ou une langue qui 
La néographie dans les pratiques langagières des jeunes Francophones égyptiens sur Facebook

ressemblait à la langue russe mais il a constaté par hasard que c'était un langage adopté de la plupart des jeunes. Ainsi, il s'est demandé la raison de l'emploi de cette néographie bizarre et pourquoi les jeunes n'adoptent pas l'alphabet standard de la langue. Selon lui, si les jeunes justifient leur choix pour ce langage par des raisons techniques ou informatiques, pourquoi alors ils n'écrivent pas des termes et des expressions complètes en anglais ? Pour lui, c'est le langage des analphabètes qui ne maîtrisent ni le français ni l'anglais ni l'arabe et par conséquent c'est le langage de celui qui n'a aucune connaissance parfaite de la langue ${ }^{39}$.

Les jeunes sont fascinés par l'emploi du rébus dans leurs pratiques langagières et parfois ils échangent des mots à la manière des puzzles linguistiques dans l'acte communicatif:

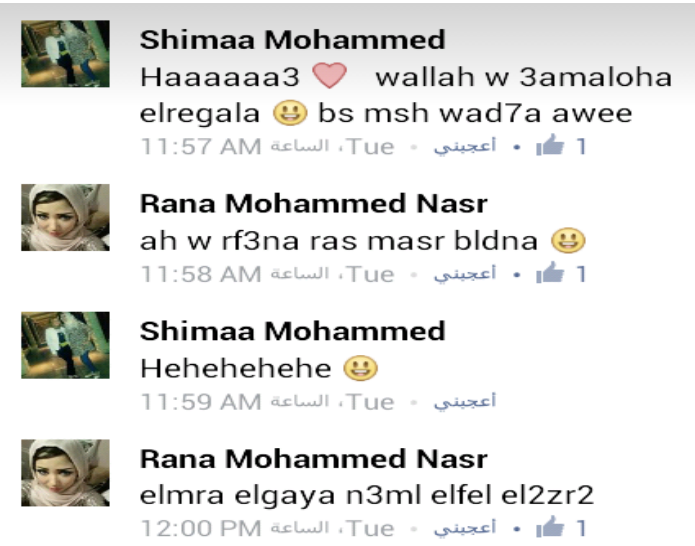

Nous constatons que ce langage n'a pas de règles fixes et les locuteurs écrivent à leur gré sans aucun contrôle. Il nous paraît que ces jeunes utilisent ce langage énigmatique pour échapper aux contraintes sociales, aux obligations familiales et ils essayent d'imposer une norme linguistique dans le monde technologique virtuel comme le dit Abdeljalil Bennoui :"Donc, à travers l'usage de la néo-orthographe, les internautes désirent être identifiés par la société comme un groupe particulier. Cela peut correspondre au verlan dans la langue orale, apparu dans les banlieues françaises comme étant un langage crypté spécifique aux jeunes. ${ }^{40 " .}$ 


\section{Les émoticônes}

Les émoticônes sont considérées comme de petits symboles formés d'une suite de caractères alphanumériques qui sont utilisées dans un message électronique pour former un visage stylisé exprimant une émotion, une humeur ou une appréciation qui représente un trait physique, une action ou un personnage ${ }^{41}$. Les émoticônes indiquent les émotions et les attitudes subjectives des locuteurs dans un cadre gestuel et mimique. Il s'agit d'un signe qui, d'après le point de vue sémiotique, s'inscrit entre la langue et le geste et qui remplit à peu près les mêmes fonctions des interjections ${ }^{42}$. Selon Yves et Marcoccia, la première fonction de l'émoticône consiste à jouer à l'écrit le rôle que jouent les mimiques faciales ${ }^{43}$. Cosnier $^{44}$ met l'accent sur la fonction phatique qui établit une communication continue des énoncés. Marrcoccia observe que les émoticônes peuvent remplir trois autres fonctions essentielles : fonction expressive, interprétative, marqueur de relation et la politesse ${ }^{45}$. Dans le langage Facebook, les jeunes utilisent énormément ces figurations dans leurs conversations quotidiennes. Ces smileys traduisent le cas de l'humour, de la tristesse, de la joie des interlocuteurs. Ces formes d'expression non verbales donnent vie à la conversation et expriment aussi des sentiments implicites de la part des interlocuteurs. Le Facebook permet à ses utilisateurs de répondre à la photo ou à la publication par le moyen d'émoticône et d'autocollants. L'internaute peut facilement cliquer sur l'émoticône souhaitée pour exprimer son admiration, son adoration, sa joie, son étonnement, sa colère ou sa tristesse de la publication ou de la photo des autres. Dans ces émoticônes, nous pouvons voir des têtes qui rient, pleurent, rougissent, doutent, tirent la langue, grognent ou vomissent. Nous observons aussi des animaux, des instruments de musique, de fruits, et des activités sportives ${ }^{46}$. Ci-dessous des exemples des smileys utilisés par les jeunes dans leurs conversations quotidiennes:




Ces smileys peuvent traduire des sentiments difficiles à être exprimés par les mots et par conséquent les locuteurs les emploient pour faire passer indirectement leurs messages ou leurs commentaires aux destinataires. Parfois, l'acte de communication se fait entièrement en émoticônes sans que les locuteurs expriment en mots leurs souhaits ou leurs demandes. Voici un exemple où nous remarquons l'emploi des émoticônes qui accompagnent les commentaires des interlocuteurs:

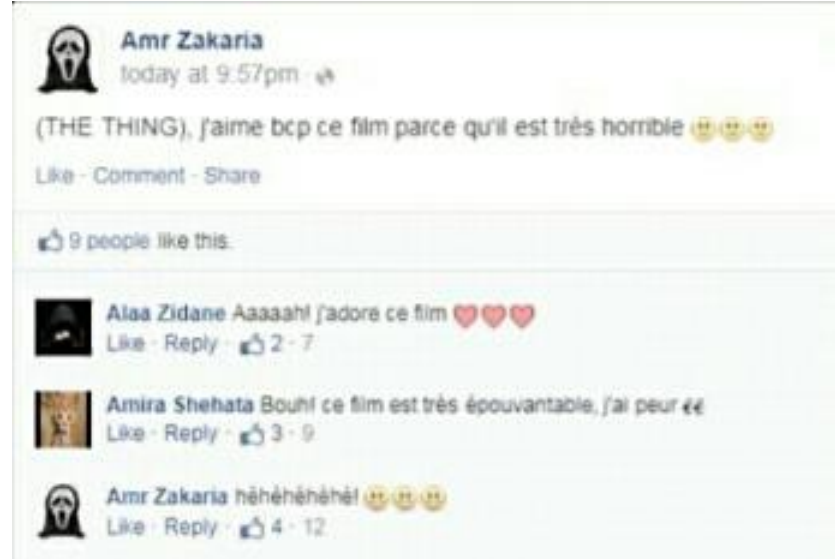

Nous observons, dans l'exemple ci-dessus, que l'emploi des émoticônes coïncide avec la nature de la publication et des commentaires. Le locuteur qui a rédigé la publication décrit son impression d'un film horrible qu'il a vu en finissant ses mots par une émoticône qui traduit ses sentiments. Le premier interlocuteur, qui répond a la publication, insère le signe du cœur qui concorde parfaitement avec le verbe adorer utilisé par lui. Le dernier interlocuteur exprime aussi sa joie et son rire en mettant le signe d'un visage souriant. L'emploi de ces émoticônes n'est pas limité aux discussions en français mais nous l'observons également dans toutes les interactions des internautes en toutes langues. Ces émoticônes, qui accompagnent les énoncés, fortifient le pouvoir des mots et peuvent transmettre des sentiments d'une manière implicite, ce qui indique que les énoncés et les émoticônes sont homogènes et tous les deux échangent la fonction interprétative dans un acte de communication où se mêle l'image et la langue. Bref, les émoticônes remplissent le même message que les énoncés mais d'une manière brève, décryptée, et rapide. Elles ponctuent nos journées, impriment nos humeurs, 
confessent nos sentiments intimes, les émoticônes sont partout ${ }^{47}$ et nous pouvons absolument tout dire de la manière que nous souhaitons. Ces émoticônes imitent les mimiques faciales comme à l'oral et elles aident le locuteur à exprimer subjectivement ses attitudes et à bien performer ses émotions.

\section{-Les onomatopées}

L'onomatopée est la création d'un mot dont le son suggère celui de la chose qu'il dénomme ${ }^{48}$. Les onomatopées sont des mots qui imitent phonétiquement, d'une manière approximative, certains sons ou certains bruits comme les cris des animaux, les bruits des machines, les sons des instruments de musique. Dans le langage des jeunes francophones égyptiens sur Facebook nous observons souvent ce phénomène; les jeunes peuvent tout simplement dire, hhhhh, hahaha, pour exprimer le rire ou le sourire, et ils peuvent écrire ohhh pour l'exclamation, et ouf pour exprimer le soulagement ou la colère, ohhh pour l'étonnement. Ces onomatopées sont écrites soit en français soit en arabe, soit en anglais; ça dépend du niveau linguistique des locuteurs qui échangent des mots et des expressions dans les discussions instantanées ou dans des commentaires pour des publications publiques. Bref "d'une manière plus immédiate, les onomatopées sont faites à partir de sons ou du bruit, interprétés d'ailleurs différemment selon les langues ${ }^{49 " .}$

\section{Les étirements graphiques}

Les pratiques langagières des jeunes sur les réseaux sociaux en général et sur Facebook en particulier se caractérisent par les étirements graphiques qui résident dans le fait de la démultiplication de certaines voyelles et consonnes en rendant le mot sous une forme trop longue. Les locuteurs font allonger la graphie des mots pour transcrire l'oralité et pour imiter la langue écrite pour y faire passer des sentiments et des émotions pour les destinataires. Ces étirements graphiques sont spécifiques au langage des discussions instantanées parce qu'elles exigent la souplesse du clavier d'un ordinateur ${ }^{50}$ ou d'un portable. Voici des exemples : 
La néographie dans les pratiques langagières des jeunes Francophones égyptiens sur Facebook
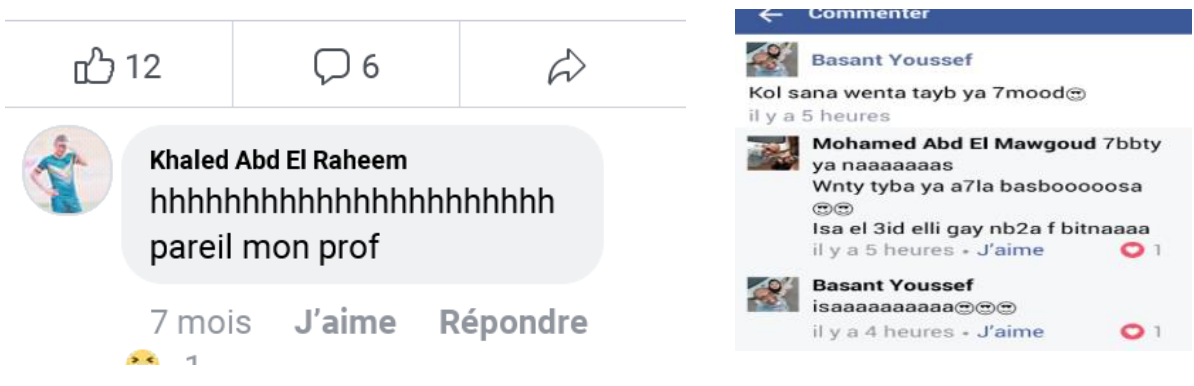

Dans les deux exemples mentionnés ci-dessus, nous observons que les locuteurs démultiplient plusieurs fois des consonnes et des voyelles. Dans le premier exemple écrit en français, c'est le $h$ qui est répété pour plus de vingt fois. Dans le deuxième exemple écrit en arabe en caractères latins, nous remarquons une alternance des étirements graphiques par les deux interlocuteurs, la locutrice répète la voyelle $o$ puis le locuteur allonge les voyelles a et o, puis la locutrice répond en répétant la voyelle $a$ pour plus de huit fois. Nous déduisons de l'emploi des étirements graphiques, soit en français soit en arabe, que les locuteurs essayent d'imiter la langue orale par le prolongement graphique de certaines lettres des mots, ce qui fait du dialogue "le lieu privilégié d'une expression difficile ${ }^{51 "}$. Le dialogue stimule les locuteurs à faire tout le possible pour imiter la langue orale de tout ce qu'elle porte " des répétitions, interruptions, recherches de mots, qu'on peut comparer aux ratures d'un brouillon ${ }^{52 " . ~ P a r ~ l ' e m p l o i ~ d e s ~}$ étirements graphiques, nous constatons également que la langue écrite n'arrive pas à faire fidèlement passer les émotions si le locuteur adopte uniquement la forme classique des mots.

De plus, la ponctuation n'est pas considérée seulement "un système des signes permettant de séparer les éléments et les phrases d'un texte écrit, d'indiquer la structure et de noter certaines intonations ${ }^{53 "}$ mais ces signes ont une valeur expressive, émotive et affective par la démultiplication du même signe ${ }^{54 "}$. Le locuteur peut dire facilement comment ça va ??????? Tu me manques!!!!!!!!! de cette manière et il peut démultiplier les points d'interrogation et d'exclamation à sa guise. Ici La démultiplication des signes d'interrogation et d'exclamation cache des sentiments chaleureux et des demandes pressantes de la part des interlocuteurs grâce à la valeur émotive et expressive de la ponctuation expressive. 
D'autre part, nous constatons que le locuteur emploie parfois la majuscule pour faire passer des messages implicites au destinataire et il peut écrire soit énoncé complet, soit un seul mot dans l'énoncé est mis en évidence. Dans le langage Facebook, nous trouvons l'emploi fréquent de ce phénomène chez les jeunes égyptiens en général et chez les francophones en particulier. Voici deux exemples rédigés en français:
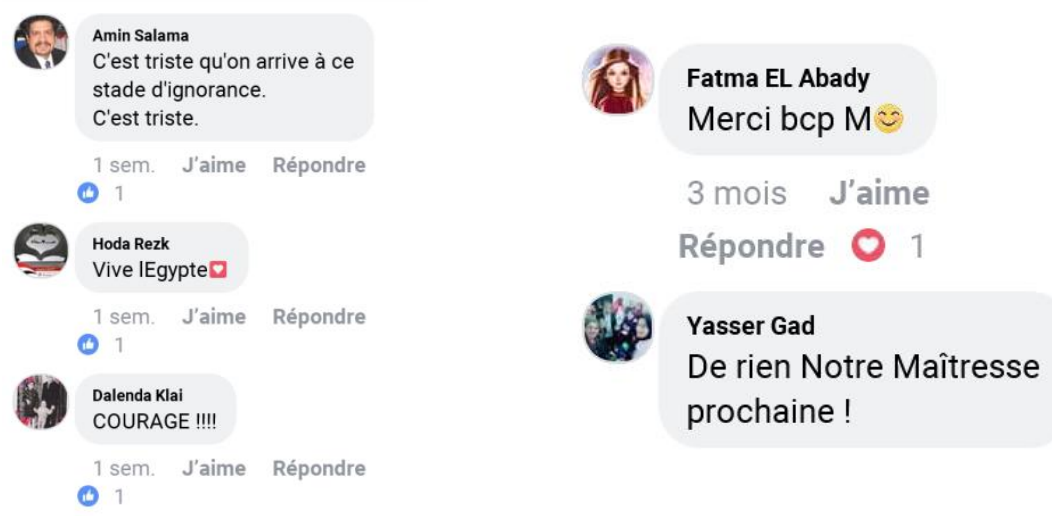

Dans la première photo, nous observons que le locuteur écrit en majuscule le mot Courage, tandis que dans la deuxième photo c'est l'emploi de la majuscule dans la lettre $N$ de l'adjectif possessif Notre et la lettre $M$ du mot Maitresse. De cette façon, nous comprenons que cette mise en relief des quelques mots ou de quelques lettres en majuscule représente une majuscule de signification qui est trop chargée de sens et qui s'oppose à la majuscule de position qui ne s'intéresse qu'à la première lettre du mot . Donc l'emploi des majuscules n'est jamais par hasard, mais par cet emploi les locuteurs francophones veulent mettre l'accent sur les mots les plus importants dans l'énoncé puisqu'ils font passer des émotions partagés par les mots choisis et qui remplacent parfois l'intonation dans les discussions orales des interlocuteurs.

\section{Le verlan et l'altération consonantique}

Le verlan est un procédé argotique qui consiste à inverser les syllabes de certains mots, de certaines locutions. C'est un phénomène productif mais aussi typé et difficilement identifiable. Le verlan représente le langage de la rue et de la banlieue et le langage des quelques programmes des jeunes, de quelques films et des chansons. 
La néographie dans les pratiques langagières des jeunes Francophones égyptiens sur Facebook

Voici des exemples du verlan utilisés par les francophones:

\begin{tabular}{|l|l|}
\hline Le mot en français & Le mot verbalisé \\
\hline Merci & cimer \\
\hline Noir & Renoi \\
\hline Lourd & Relou \\
\hline Femme & Meuf \\
\hline Rigoler & Golerie \\
\hline
\end{tabular}

Nous pouvons dire que l'emploi du verlan dans les discussions instantanées des francophones égyptiens est assez rare et nous n'en relevons qu'un seul exemple pour le mot femme qui se transforme en meuf:

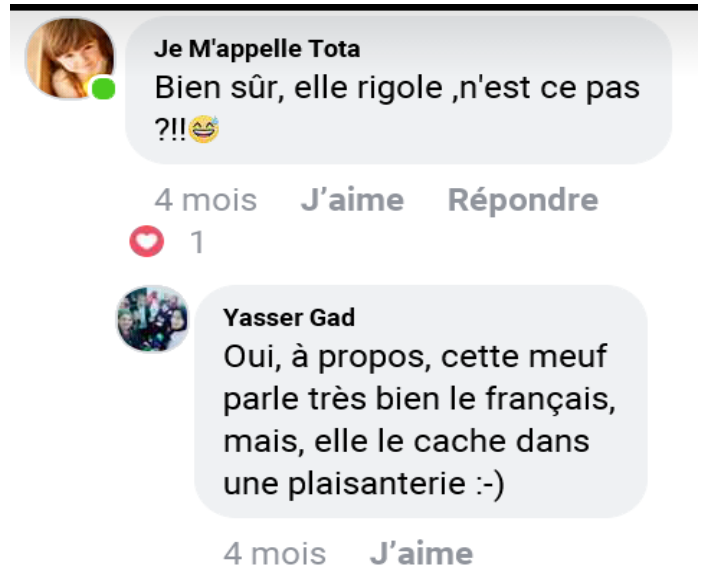

Dans l' exemple ci-dessus, nous remarquons que le mot femme devient meuf, ce qui représente une menace directe de la langue et de l'avenir linguistique des apprenants. D'autre part, quand les locuteurs francophones égyptiens changent le code linguistique vers l'arabe sur Facebook, ils adoptent un autre phénomène qui déforme la forme du mot. L'altération consonantique représente un phénomène très dangereux pour l'avenir de la langue parlée ou écrite puisqu'une telle substitution menace la structure interne et fait disparaitre rapidement toute production littéraire ou scientifique de la langue. Les jeunes égyptiens remplacent dans leurs discussions instantanées des consonnes de la langue maternelles par d'autres inventées et cela ne fait que peser le fardeau linguistique que subit la langue arabe à cause des jeunes 
cherchant par tous les moyens à la mode et les pratiques langagières imitées et irraisonnées. Ce phénomène de mutation consonantique réside dans la substitution d'une consonne à une autre et l'articulation du mot devient par conséquent différente de la prononciation originale et le statut de l'alphabet arabe devient opaque à cause de cette déformation intentionnée de la part des locuteurs jeunes. Ceux-ci remplacent quelques consonnes de l'alphabet arabe par d'autres qui sont proches en prononciation ${ }^{55}$ mais ça peut créer un cas de chaos linguistique. Le verlan et l' altération consonantique représentent une alarme pour les puristes de la langue car l'abus de l'emploi de la part de quelques locuteurs engendrera sûrement une influence négative sur l'orthographe pour les nouveaux apprenants de la langue et pour les futurs enfants dont les pères déforment à leur gré l'alphabet de leur propre langue.

\section{Conclusion}

De tout ce qui précède, nous pouvons résumer quelques remarques sur les pratiques langagières des jeunes égyptiens sur Facebook :

- la néographie représente un instrument d'expression rapide entre les jeunes puisque dans le monde virtuel ils écrivent et lisent rapidement le contenu du message et ils vont à l'essentiel tout directement $^{56}$, ce qui leur permet de surmonter les contraintes linguistiques de la situation de la communication et ils peuvent échanger rapidement et librement des codes linguistiques communs et admis par tous les locuteurs de la même catégorie sociale ;

- la néographie menace l'avenir de la langue: nous avons observé que les jeunes déforment la langue maternelle et la langue d'étude par l'emploi de nouvelles formes linguistiques comme le rébus typographique et l'alternance codique et aussi l'emploi des étirements linguistiques puis la mutation consonantique. Toutes ces particularités lexicales et graphiques dans le langage des jeunes peuvent nuire inconsciemment à leurs compétences orthographiques et par conséquent cela influencera négativement le statut futur de l'orthographe normative de la langue. Pour cela l'Académie française et arabe doivent pratiquer des efforts considérables pour améliorer le statut actuel de la langue et l'état doit imposer des lois fermes pour 
La néographie dans les pratiques langagières des jeunes Francophones égyptiens sur Facebook

sauvegarder la langue.

- la néographie est une forme qui montre l'imperfection linguistique. Les jeunes emploient des codes hybrides formés de français, d'arabe, ou d'anglais, ce qui signifie que les interlocuteurs ne peuvent pas souvent échanger réciproquement en une seule langue d'une manière parfaite comme le soulignait auparavant Ahmed Khaled Tawfik en affirmant que ce langage appartient aux illettrés qui ne maîtrisent aucune langue.

- la néographie représente une composante identitaire et sociale. Ces pratiques langagières des jeunes utilisent des codes linguistiques propres et admis par tous les membres de la communauté linguistique, et cela indique qu'il y a une norme identitaire dans le monde virtuel et ce langage représente une rébellion sociale de la part des jeunes à l'égard des contraintes sociales. Bref les pratiques langagières des jeunes sur Facebook est un bon indice de la faiblesse du statut de la langue chez les locuteurs et les efforts modestes des écoles puis des universités pour la sauvegarder de toutes les tentatives de déformations linguistiques potentielles chez les jeunes qui sont fascinés d'imiter et d'appliquer n'importe quelle nouveauté linguistique même si cela est aux dépends de la langue nationale. Bref la langue doit être "observée, étudiée, comprise, et apprivoisée, si l'on veut éviter que le monde ne se transforme pas en jungle, si l'on veut éviter que l'avenir ne ressemble aux pires images du passé $e^{57 " \text {. }}$ 


\section{$\underline{\text { Notes }}$}

${ }^{1}$ Cf., A.DEJOND La cyberl@ngue francaise, Paris, La Renaissance du Livre, 2002, Cf., Jacques ANIS, communication électronique scripturale et formes langagières, Actes des Quatrièmes rencontres réseaux Humains, Réseaux technologiques

${ }^{2}$ Abdelrahaman SELIM, le nombre des utilisateurs de Facebook en Egypte arrive à 38 million, le quotidien Alwattan, communication et technologie, 6 mars, 2018

${ }^{3}$ Dans cette partie, je me réfère à l'article de Madiha DOSS, Vincent BATTESTI, $\underline{\text { Les }}$ pratiques linguistiques et leurs contextes sociaux. Vincent Battesti; François Ireton. L'Égypte au présent, Inventaire d'une société avant révolution, Éditions Sindbad, ActesSud, La Bibliothèque arabe, Hommes et Sociétés pp.971:993, 2011.

${ }^{4}$ Ibid., P. 971

5 Cf., $\underline{\text { Ibid, }}$, p.973

${ }^{6}$ Ibid. p.972

${ }^{7}$ Cf., Ibid, p.973

${ }^{8}$ Ibid.

${ }^{9}$ Catherine MILLER, Les Saidis au Caire: accommodation dialectale et construction identitaire, maison Neuve et Larousse, 2005, PP:181

${ }^{10}$ Cf., A.DEJOND, Op.cit., p.34

${ }^{11}$ Jacques ANIS, Internet, communication et langue francaise, Paris, Hermès Science Publications, 1999, p.31

${ }^{12}$ Jan LAZAR, quelques remarques sur le discours électronique médie: de la graphie vers la morphosyntaxe, université d'Ostrava: http://www.atilf.fr/IMG/pdf/seminaires/Seminaire_sms_resume_Lazar_2012-04-26.pdf, P.2

${ }^{13}$ Ibid

${ }^{14}$ Cf., Hoda QAZ', Le langage des jeunes dans les universités: réalité et espoir, revue Oud - انظر : هدى القزع، لغتة الشباب بين الواقع والمأمول، مجلة عود

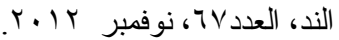

${ }^{15}$ Directeur du centre des sciences du langage à l'Université libanaise

${ }^{16}$ Cf.,Ghassan Mourad: humanités numériques: apprivoisement de la langue pour le traitement automatique et des question en culture technologiques pour la création de nouvelles sciences humaines, Bayrût, Dar Almatbouaat,2013

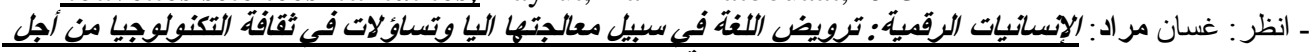

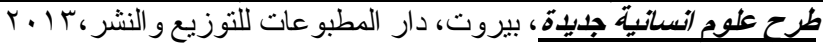

${ }^{17}$ Expert au centre national des recherches sociales et pénales en Egypte

${ }^{18}$ Cf., Ali Salah MAHMOUD , la culture de la jeunesse arabe: a-t-elle des particularités propres? , 8ème colloque: Questions de la jeunesse au début de XXIe siècle, 23-25 mai 2006, centre national des recherches sociales et penales,2006, p.33:63.

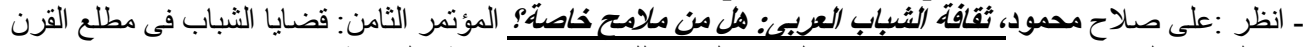

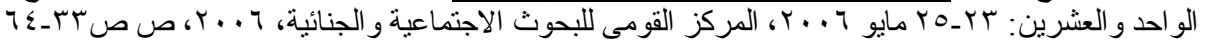

${ }^{19}$ Cf., Nahla EINEMR, Phénomène d'arabe hybride touche la langue d'une monstruosité linguistique, le quotidien $\mathrm{Al}$ Wafd, mardi 19 janvier 2016.



20 Richard PEEL, L'internet et l'utilisation des langues, une études de cas dans les Emirats-unis, international journal on multicultural Societies, Vol.6, No.1, 2004, p.171..

21 H.ATIFI, la variation culturelle dans les communications en ligne: analyse ethnographique des forums de discussions marocains, langage et société, 2003, 
104,57:82, cité par: Ouardia ACI, Facebook : pratiques langagières et discours sur les langues en Algérie, KEBBAS M. et KARA A. Y., (2014), revue SOCLES n³, laboratoire LISODIP, ENS de Bouzareah, pp. 21

${ }^{22}$ Ibid.

${ }^{23}$ Jean DUBOIS et al., Dictionnaire de linguistique, Larousse, 2002, p.496

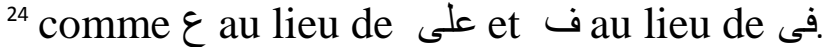

${ }^{25}$ Jean DUBOIS et al, $\underline{\text { Op.cit. }}$. P.13.

${ }^{26}$ Catherine DELAMARCHE et al., Hachette, Dictionnaire de la langue francaise, sous la direction d'Amiel (Philippe), Paris, Hachette, 1994, p.4.

${ }^{27}$ Remi BOVE, Etude de quelques problèmes de phonétisation dans un système de synthèse de la parole à partir de SMS, In Actes de RECITAL 2005, Dourdan, p.22

${ }^{28}$ John GUMPERZ, Engager la conversation, Paris, Edition de minuit, 1989, p.72

${ }^{29}$ Abdelfattah NISSABOURY., Arabophones et francophones du Maroc : un bilinguisme dynamique, in Revue québécoise de linguistique, 1999, pp : 69-87.

${ }^{30}$ Chantal CHARNET, «Ana je pose la question » ou "quelques facons de parler de locuteurs marocains : étude de manifestation du contact linguistique $»_{2}$ in MANZANO, Les Langues du Maghreb et du sud méditerranéen, Rennes, P.U.R, 1999.

${ }^{31}$ Robert LAFONT, Quarante ans de sociolinguistique à la périphérie, Paris, l'Harmattan, 1997

${ }^{32}$ John GUPMER, Op.cit., p.73

33 Cf., Yasmina BOUTAGHANE, l'alternance codique dans les medias algeriens, cas: $l^{\prime}$ emission televisee le Grand Sbitar, thèse de Master, Université Université Abderrahmane mira-Bejaia, 2016, p.12.

${ }^{34}$ Shan POPLACK, Conséquences linguistiques du contact de langues : un modèle d'analyse variationniste, in, Langage et société, 1988, in MARAOUI Lynda, Etude sociolinguistique de l'alternance des langues dans les débats télévisuels à caractère sportif en Algérie, Université Université Abderrahmane mira-Bejaia, 2017, p.24.

${ }^{35}$ Yasmina BOUTAGHANE, $\underline{\text { Op. } \text {. Cit. }}$. , P.13

${ }^{36}$ Catherine DELAMARCHE et al., Op.Cit., p934

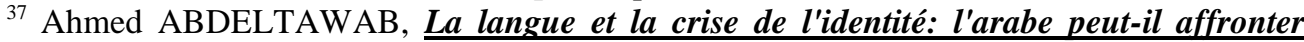
l'invasion numérique et la néographie? Revue Loughat Al-Asr- Al- Ahram, février 2019.

"هى لغة غير محددة القو اعد مستحدثة وغير رسمية ظهرت كنتيجة طبيعية للثورة الرقمية التى يشهدها المجتمع منذ عدة سنو ات لهن

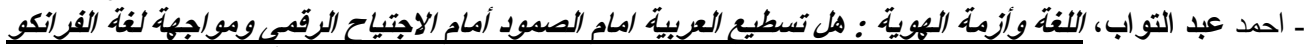

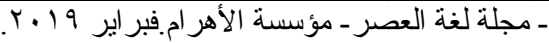

${ }^{38}$ Kahina ISSAADI, la créativité lexicale chez les jeunes algériens sur les réseaux sociaux, Université Abderrahmane- Mira Bejaia, 2015, p.52

${ }^{39}$ Cf., Ahmed khaled TAWFIK, l'arabe hybride (franco-arabe), le quotidien Al Etihad, Les Emirats-unis, mai, 2013.

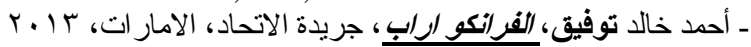

${ }^{40}$ Abdeldjalil BENNOUI, Les représentations de la néo-orthographe chez les internautes constantinois, Revue Expressions, $\mathrm{n}^{\circ} 1$, Juin 2015, p.71

${ }^{41}$ Le Grand Robert de la langue francaise , version électronique, 2eme édition, sous la direction de Alain Rey, 2005 
${ }^{42}$ Pierre HALTE, Les marques modales dans les chats :étude sémiotique et pragmatique des interjections et des émoticônes dans un corpus de conversations synchrones en ligne, thèse de doctorat, Université de Lorraine, 2013, p.4

${ }^{43}$ Ibid., p.42

44 J.COSNIER, les gestes du dialogue, la communication non verbale, in le Dialogue, psychologie de la motivation, n 21, Lyon, Chronique sociale, PP:129:138, in Pierre HALTE, Op.cit., p.43.

${ }^{45} \mathrm{Cf} ., \mathrm{M}$. MARCOCCIA, les smileys, une représentation iconique des émotions dans la communication mediatisee par ordinateur, in les Emotions dans les interactions, Lyon, Presse Universitaires de Lyon, 2000, in P.HALTE, $\underline{\text { op.Cit. }}$, p. 42

46 MARIE VATON, Parlez-vous “emoji”? Tendances, N 2614, 11-122014,http://www.sud4science.org/?q=fr/system/files/nouvel-obs-11-12-2014.pdf

${ }^{47}$ Ibid.

${ }^{48}$ Catherine DELAMARCHE et al., $\underline{\text { Op. } \text { Cit., p.762 }}$

${ }^{49}$ Jean DUBOIS et al., Op.Cit.., p.167

${ }^{50}$ Jacques ANIS, $\underline{\text { Op. } \text { Cit. }}$., p. 8

${ }^{51}$ Françoise Rullier THEURET, le dialogue dans le roman, Paris, Hachette, 2001, p. 92

${ }^{52}$ Ibid.

${ }_{53}$ Catherine DELAMARCHE et al, Op.Cit., p. 859

${ }^{54}$ M. MARCOCCIA, $\underline{\text { op. Cit. }}$., P.2

55

\begin{tabular}{|c|c|c|}
\hline Le mot original & Le mot invente & Le sens en français \\
\hline حقيقة & حكيكة & verité \\
\hline طبعا - طبا & تبعا & Absolument oui \\
\hline سيایى & زيدى. & monsieur \\
\hline عل عل عل & عثل & Miel (mignon) \\
\hline صغير | | صغير & صغنن & petit \\
\hline جميلة & كميلة & joli \\
\hline صباح الخير & ثباح الخير & bonjour \\
\hline صاحبى & ساحبى & Mon ami \\
\hline
\end{tabular}

${ }^{56}$ A.DEJOND, $\underline{\text { Op.Cit. }}$. p.34

${ }^{57}$ A.MAALOUF, les identités meurtrières, Paris, Grasset, 1998, p.33 
La néographie dans les pratiques langagières des jeunes Francophones égyptiens sur Facebook

Bibliographie consultée

- Abdelfattah NISSABOURY, Arabophones et francophones du Maroc : un bilinguisme dynamique, in Revue québécoise de linguistique, 1999

- A.DEJOND, La cyberl@ngue francaise, Paris, La Renaissance du Livre, 2002

- Abdeldjalil BENNOUI, Les représentations de la néo-orthographe chez les internautes constantinois, Revue Expressions, $\mathrm{n}^{\circ} 1$, Juin 2015

- A.MAALOUF, les identités meurtrières , Paris, Grasset, 1998

- Catherine MILLER, Les Saidis au Caire: accommodation dialectale et construction identitaire, maison Neuve et Larousse, 2005

- Chantal CHARNET, «Ana je pose la question » ou « quelques facons de parler de locuteurs marocains : étude de manifestation du contact linguistique ", in MANZANO, Les Langues du Maghreb et du sud méditerranéen, Rennes, P.U.R. 1999.

- Françoise Rullier THEURET, le dialogue dans le roman, Paris, Hachette, 2001

- H.ATIFI, la variation culturelle dans les communications en ligne: analyse ethnographique des forums de discussions marocains, langage et société, 2003

Jacques ANIS, Internet, communication et langue francaise, Paris, Hermès Science Publications, 1999.

- Jacques ANIS, communication électronique scripturale et formes langagières, Actes des Quatrièmes rencontres réseaux Humains, Réseaux technologiques, Poitiers, Université de Poitiers, 2006: http://rhrt.edel.univpoitiers.fr/document.php?id=547.

- Jan LAZAR, quelques remarques sur le discours électronique Médie: de la graphie vers la morphosyntaxe, université d'Ostrava

:http://www.atilf.fr/IMG/pdf/seminaires/Seminaire_sms_resume_Lazar_2012 -04-26.pdf

- John GUMPERZ, Engager la conversation, Paris, Edition de minuit, 1989

- Lynda MARAOUI, Etude sociolinguistique de l'alternance des langues dans les débats télévisuels à caractère sportif en Algérie, Université Université Abderrahmane mira-Bejaia, 2017

- Madiha DOSS, Vincent BATTESTI, Les pratiques linguistiques et leurs contextes sociaux. Vincent Battesti; François Ireton. L'Égypte au présent, Inventaire d'une société avant révolution, Éditions Sindbad - Actes-Sud, La Bibliothèque arabe, Hommes et Sociétés pp.971-993, 2011. 
- Marie VATON, Parlez-vous “emoji”? Tendances, No 2614, 11-122014,http://www.sud4science.org/?q=fr/system/files/nouvel-obs-11-12-

2014.pdf

- Michel MARCOCCIA, les smileys, une représentation iconique des émotions dans la communication médiatisée par ordinateur, in les Emotions dans les interactions, Lyon, Presse Universitaires de Lyon, 2000.

- Ouardia ACI, Facebook : Pratiques langagières et discours sur les langues en Algérie, revue SOCLES n³, laboratoire LISODIP, ENS de Bouzareah, 2014.

- Pierre HALTE, Les marques modales dans les chats :étude sémiotique et pragmatique des interjections et des émoticônes dans un corpus de conversations synchrones en ligne, thèse de doctorat, Université de Lorraine, 2013

- Remi BOVE, Etude de quelques problèmes de phonétisation dans un système de synthèse de la parole à partir de sms, In Actes de RECITAL, Dourdan, 2005

- Richard PEEL, L'internet et l'utilisation des langues, une études de cas dans les Emirats-unis, international journal on multicultural Societies, Vol.6, No.1, 2004

- Robert LAFONT, Quarante ans de sociolinguistique à la périphérie, Paris, l'Harmattan, 1997

- Shan POPLACK, Conséquences linguistiques du contact de langues : un modèle d'analyse variationniste, in, Langage et société, 1988

- Dictionnaires

- Catherine DELAMARCHE et al., Hachette, Dictionnaire de la langue francaise, sous la direction d'Amiel (Philippe), Paris, Hachette, 1994

- Jean DUBOIS et al., Dictionnaire de linguistique, Larousse, 2002, p.496.

Thèses consultées

Kahina ISSAADI, la créativité lexicale chez les jeunes algériens sur les réseaux sociaux, Université Abderrahmane- Mira Bejaia, 2015

- Yasmina BOUTAGHANE, l'alternance codique dans les médias algériens, cas: l'émission télévisée le Grand Sbitar, thèse de Master, Université Abderrahmane mira-Bejaia, 2016

- Zeinab BETTICHE, l'influence du cyberlangage sur le francais normatif chez les jeunes algeriens, Université Larbi Tebessa, 2016 
La néographie dans les pratiques langagières des jeunes Francophones égyptiens sur Facebook

Des références arabes:









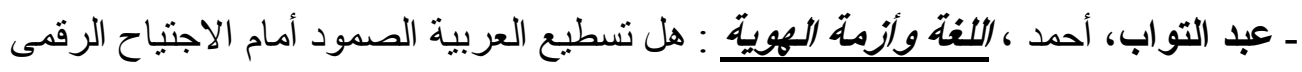

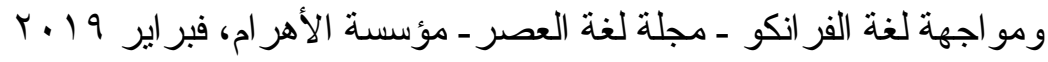

ـ محمود، على صلاح ، ثقافة الثباب العربح: هل من ملامح خاصة؟؟ المؤتمر الثامن: قضايا



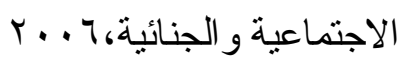

- مر اد، غسان: الإسانيات الرقمية: ترويض اللغنة في سبيل معالجتها اليا وتساؤلات في ثقاقة التكنولوجيا من أجل طرح علوم انسانية جبيةة، بيروت، دار المطبوعات للتوزيع






\section{الكتابة المستحدثة فى الممارسات اللغوية لناطقى الفرنسية فى مصر



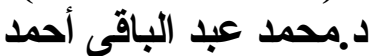

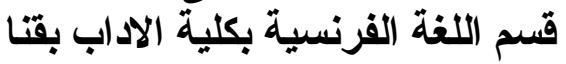 \\ جامعة جنوب الوادى الون الادي}

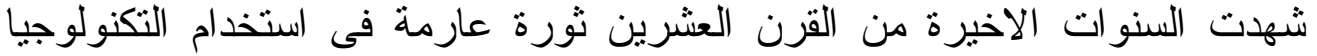



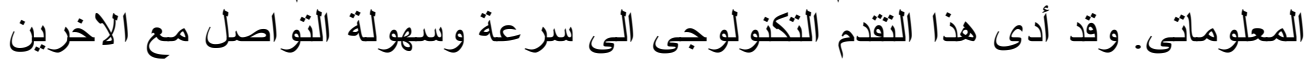

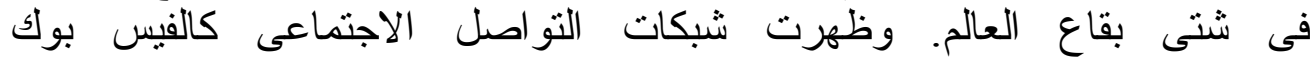

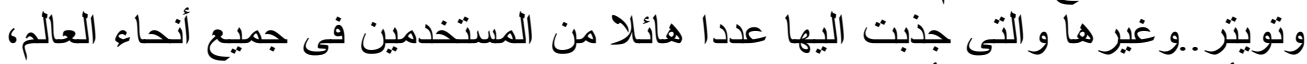



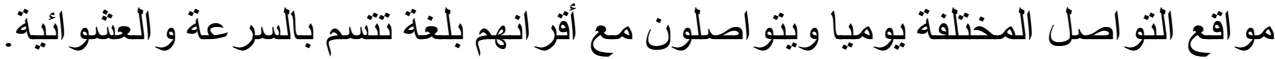

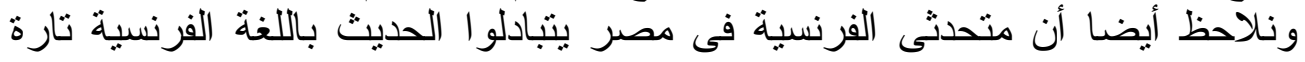

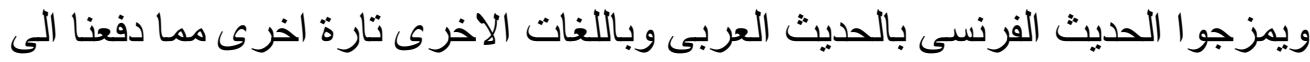

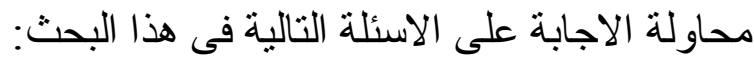

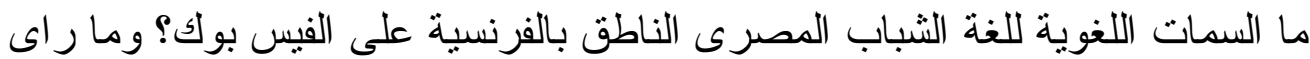

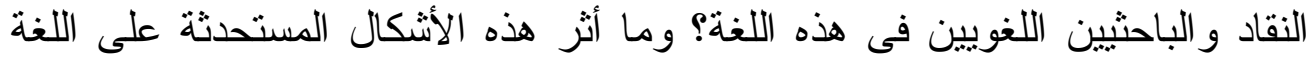

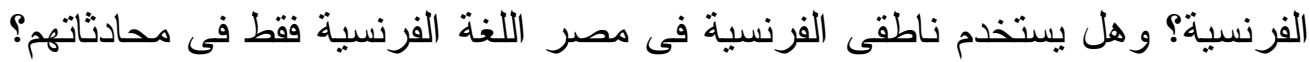

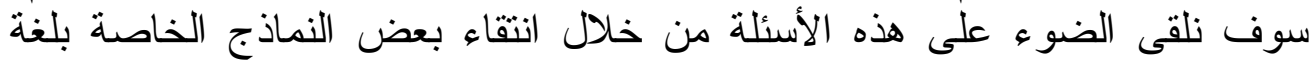

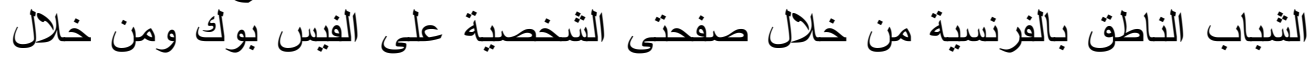

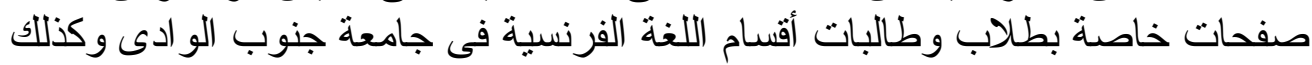

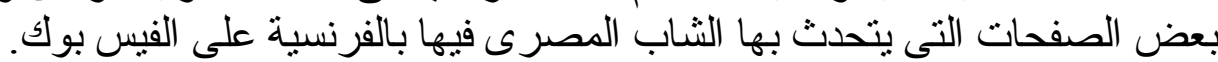

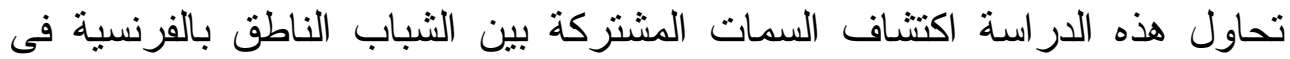

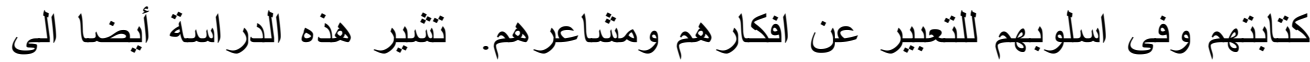



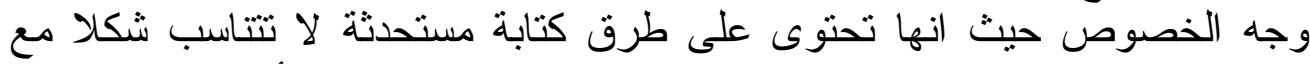

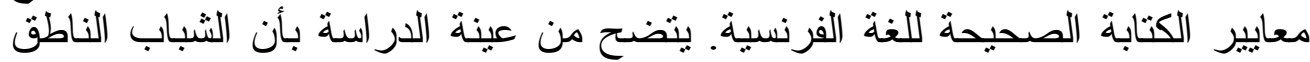

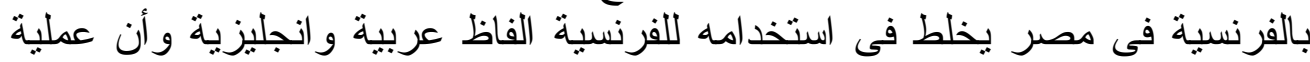

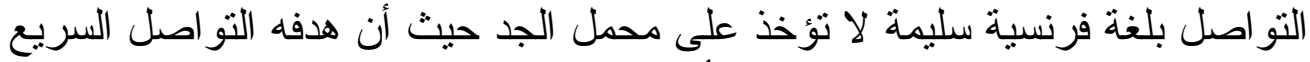

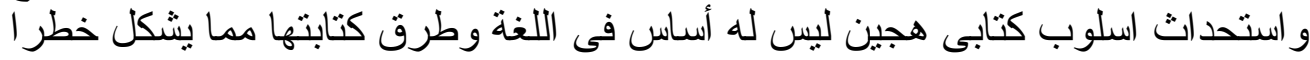

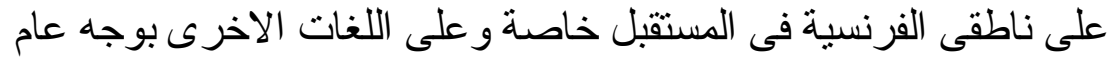

